\title{
CHUVA E PRODUTIVIDADE DA SOJA NA FAZENDA EXPERIMENTAL GRALHA AZUL DA PUCPR
}

\author{
Accumulated rainfall versus soybean productivity in the Gralha \\ Azul Experimental Farm - PUCPR
}

Roberto Fendrich*

\section{Resumo}

O objetivo do presente estudo foi determinar a influência da precipitação pluvial na produtividade da soja, nas condições ambientais da Fazenda Experimental Gralha Azul da PUCPR, visando a avaliar a pertinência, ou não, de implantação de irrigação para se atingir maior produtividade da cultura.

Palavras-chave: Soja, Chuva, Produtividade da Cultura.

\section{Abstract}

The objective of the present study was to determine the influence of the pluvial precipitation to soybean productivity in the environmental conditions of the Gralha Azul Experimental Farm - PUCPR, aiming to evaluate the necessity, or not, of irrigation to reach greater crop productivity.

Keywords: Soybean, Rain, Crop productivity.

* Professor Titular do Curso de Engenharia Civil da PUCPR. Professor Adjunto do Departamento de Hidráulica e Saneamento - DHS da UFPR.

Pontifícia Universidade Católica do Paraná - PUCPR, Rua Imaculada Conceição, n. ${ }^{\circ} 1155$, Prado Velho, Curitiba - PR, CEP 80215901.

E-mail: intec@rla13.pucpr.br 


\section{Introdução}

São várias as atividades econômicas que constituem o complexo agroalimentar, destacando-se entre elas a cadeia agroindustrial da soja. O setor produtivo é a essência de toda cadeia, pois é a produção que movimenta e interliga todos os demais segmentos. A cadeia agroindustrial da soja brasileira sempre foi considerada um exemplo de sucesso no mercado mundial.

O crescimento da produção e do esmagamento da soja, na década de 70, foi tão rápido que colocou o Brasil como o primeiro exportador mundial do farelo de soja, tendo perdido recentemente essa posição para a Argentina, e é o segundo exportador mundial de soja em grão.

Uma das principais causas da variação da produtividade da soja no Brasil tem sido a ocorrência de déficit hídrico. Pela Figura 1, pode-se observar quedas na produtividade média da soja no Brasil nas safras 1977/78, 78/79 e 85/86, com perdas de $31 \%$, 30\% e $22 \%$, respectivamente, causadas pela deficiência hídrica naqueles anos.

FIGURA 1 - Produtividade média e área cultivada com soja no Brasil nas safras de 1975/76 a 1999/00

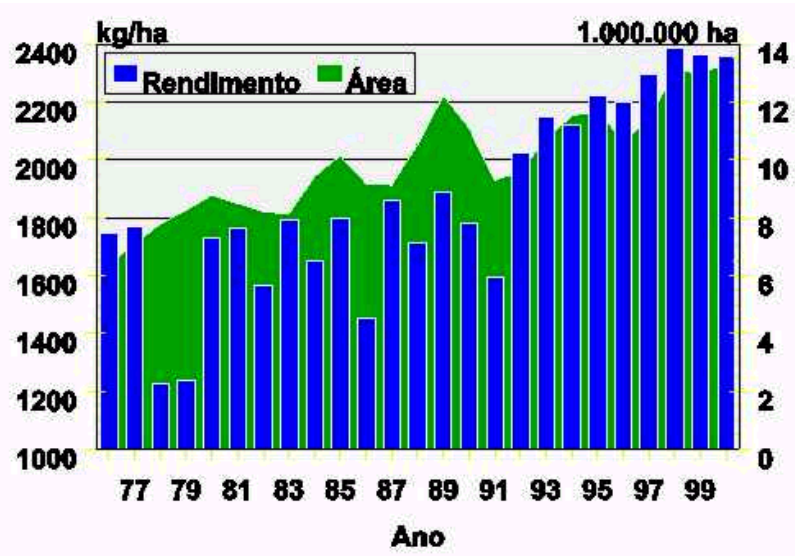

A água constitui aproximadamente 90\% do peso da planta, atuando em, praticamente, todos os processos fisiológicos e bioquímicos. Desempenha a função de solvente, por meio do qual gases, minerais e outros solutos entram nas células e movem-se na da planta. Tem ainda, papel importante na regulação térmica da planta, agindo tanto no resfriamento como na manutenção e distribuição do calor.

Para determinar as influências da chuva na produtividade da soja, o estudo objetivou ava- liar as condições da relação entre a produtividade de soja e precipitação acumulada na região do Município de Fazenda Rio Grande - PR, onde está localizada a Fazenda Experimental Gralha Azul da PUCPR, verificando a possível necessidade de implantar sistema de irrigação na cultura de soja, visando obter sua maior produtividade.

\section{Cultura da Soja}

A soja é uma leguminosa com alta capacidade de fixar nitrogênio atmosférico por simbiose, raramente tendo necessidade da suplementação de nitrogênio via adubação.

A cultura é feita em condições de clima quente nos trópicos, subtrópicos e regiões temperadas, porque a soja é bem resistente as temperaturas altas e baixas. Sabe-se, porém, que seu crescimento fica afetado por temperaturas menores que $18^{\circ} \mathrm{C}$ e maiores que $35^{\circ} \mathrm{C}$. Em algumas variedades, a floração pode atrasar se a temperatura média diária estiver abaixo dos $24^{\circ} \mathrm{C}$. Seu crescimento praticamente cessa a $10^{\circ} \mathrm{C}$, mas temperaturas do ar acima de $15^{\circ} \mathrm{C}$ já garantem sua produção comercial. Apenas 25 a 30\% das flores produzem vagens, e este número depende do vigor da planta no período do florescimento. De ano para ano, variações na temperatura média podem afetar a porcentagem de frutificação das vagens.

A planta é sensível às condições de solo saturado, este devendo ser bem drenado e arejado. O sistema radicular da soja é bem superficial. Medidas feitas indicam que cerca de $80 \%$ do seu peso se encontram na camada $0-15 \mathrm{~cm}$ e que mais de $90 \%$ se encontram na zona arável $(0-30 \mathrm{~cm})$. A raiz principal pivotante, porém, em condições favoráveis, pode alcançar profundidades maiores que $1 \mathrm{~m}$. As raízes mais profundas, apesar de em menores quantidades, parecem ter uma atividade importante. Perfis de umidade de solo indicam que até $50 \mathrm{~cm}$ de profundidade, a extração de água pela soja é muito grande, cerca de 80 a $90 \%$ da evapotranspiração real ( ETr ) vem desta camada.

Dentro de limites, a soja mostra uma notável capacidade de se adaptar a déficit de água. Ao que tudo indica, o déficit de água no período reprodutivo é o que mais afeta a produtividade. Muitas observações mostram que déficit de água, não muito severos, durante o estágio vegetativo, não afetam significativamente a produtividade. 
A demanda máxima de água, para produção máxima, varia de 450 a $700 \mathrm{~mm}$, para ciclos de 100 a 130 dias, dependendo do clima. Sua produtividade varia muito com a disponibilidade de água, adubação, espaçamento e clima. Em condições de chuva natural, boas produções são da ordem de 2,5 a $3,5 \mathrm{t} /$ ha de peso seco.

Com relação ao clima, Gomes (1990) afirma que a soja é uma planta de clima quente e úmido, uma vez que é originária do Sudeste da Ásia. Como, porém, geralmente se trata de uma planta anual, cujo ciclo vegetativo pode ser muito curto, até 80 a 90 dias, também é cultivada em climas temperados, desde que o verão seja quente e chuvoso. Faltando chuva, deverá ser irrigada. Onde o verão é curto, usam-se variedades precoces, que amadurecem antes das primeiras geadas.

Gomes (1990) coloca que, talvez, a pluviosidade ideal, para um país como o Brasil, oscile entre 1000 a $1600 \mathrm{~mm} / \mathrm{ano}$. Acredita-se que, sabendo preparar bem o solo e escolher a época de semeadura, uma pluviosidade superior a 800 $\mathrm{mm}$ seja suficiente, em se tratando de variedades cujo ciclo vegetativo não ultrapasse 120 dias. Também, acredita-se que, tomadas as devidas cautelas, sabendo escolher a data da semeadura, se possa plantar soja onde a pluviosidade seja superior a $1600 \mathrm{~mm}$. Deve-se levar em consideração que o excesso de precipitação prejudica a soja, que é relativamente resistente a períodos de seca moderados.

\section{Metodologia}

A equação genérica que relaciona precipitação pluvial acumulada versus produtividade de culturas, conforme Tubelis (1988), tem a forma:

$$
P=a+b\left(I_{\text {acum }}\right)
$$

Onde:P = Produtividade da cultura $(\mathrm{kg} /$

ha);

$\mathrm{I}_{\text {Acum }}=$ Precipitação pluvial acumulada no período da cultura $(\mathrm{mm})$;

$\mathrm{a}=$ coeficiente linear;

$\mathrm{b}=$ coeficiente angular.
O coeficiente angular "b" da Equação (1) é calculado por:

$$
b=\frac{J}{K}
$$

Onde:

$$
\begin{aligned}
& \mathrm{J}=\sum \mathrm{I}_{\text {Acum }} \times \mathrm{P}-\frac{\left(\sum \mathrm{I}_{\text {Acum }}\right) \times\left(\sum \mathrm{P}\right)}{\mathrm{N}} \\
& K=\sum\left(\mathrm{I}_{\text {Acum }}\right)-\frac{\left(\sum I_{\text {Acum }}\right)^{2}}{N}
\end{aligned}
$$

Sendo: $\mathrm{N}$ = Número de pares de valores da série Produtividade x Precipitação Pluvial Acumulada.

O coeficiente linear "a" da Equação (1) é obtido por:

$$
a=\frac{\Sigma P}{N}-b \cdot \frac{\Sigma I_{a c u m}}{N}
$$

Onde:

$\mathrm{a}, \mathrm{b}, \mathrm{P}, \mathrm{I}$ Acum e $\mathrm{N}=$ termos definidos anteriormente.

\section{Cultura da Soja na Fazenda Experimental Gralba Azul da PUCPR}

Os dados referentes à cultura agrícola da soja, na Fazenda Experimental Gralha Azul da PUCPR, estão resumidos na Tabela 1. 
TABELA 1 - Produtividade da soja na Fazenda Experimental Gralha Azul da PUCPR

Table 1 - Soybean productivity in the Experimental Farm Gralha Azul of PUCPR

\begin{tabular}{|c|c|c|c|c|c|c|}
\hline ANO & $\begin{array}{l}\text { VARIEDADE } \\
\text { (S) }\end{array}$ & PLANTIO & COLHEITA & $\begin{array}{l}\text { ÁREA } \\
\text { (ha) }\end{array}$ & $\underset{(\mathrm{kg})}{\text { TOTAL }}$ & $\begin{array}{c}\text { PRODUTI } \\
\text { VIDADE } \\
\text { (kg/ha) }\end{array}$ \\
\hline \multirow{3}{*}{ 1993/94 } & FT 10 & $29 / 11 / 93$ & Abril/94 & 25,3 & 62.339 & 2.464 \\
\hline & $\begin{array}{c}\text { FT 10 } \\
\text { (PRINCESA) }\end{array}$ & $07 / 12 / 93$ & Maio/94 & 35,0 & 103.880 & 2.968 \\
\hline & - & - & - & - & - & - \\
\hline \multirow{3}{*}{ 1994/95 } & FT ABYARA & $10-15 / 11 / 94$ & $4-7$ e $15-24 / 04 / 95$ & 42,7 & 122.165 & 2.861 \\
\hline & BR 16 & $25-27 / 11 / 94$ & $10-15 / 04 / 95$ & 21,4 & 64.970 & 3.036 \\
\hline & - & - & - & - & - & - \\
\hline \multirow{3}{*}{ 1995/96 } & BR 16 & $\begin{array}{c}23 / 11 \mathrm{e} \\
07 / 12 / 95 \\
\end{array}$ & 11 e 20/04/96 & 7,3 & 15.578 & 2.134 \\
\hline & - & - & - & - & - & - \\
\hline & - & - & - & - & - & - \\
\hline \multirow{3}{*}{ 1996/97 } & BR 16 & $27-28 / 11 / 96$ & $10-12 / 04 / 97$ & 33,8 & 72.332 & 2.140 \\
\hline & BR 16 & $28-29 / 11 / 96$ & $13-14 / 04 / 97$ & 45,0 & 100.350 & 2.230 \\
\hline & BR 16 & $02-03 / 12 / 96$ & $15-16 / 04 / 97$ & 25,5 & 53.754 & 2.108 \\
\hline \multirow{3}{*}{ 1997/98 } & BR 16 & $03-04 / 12 / 97$ & $18 / 04-03 / 05 / 98$ & 44,0 & 101.200 & 2.300 \\
\hline & BR 16 & $16 / 12 / 97$ & $23 / 04 / 00$ & 6,0 & 13.452 & 2.242 \\
\hline & - & - & - & - & - & - \\
\hline \multirow{3}{*}{ 1998/99 } & BR 35 & 11 a $13 / 11 / 98$ & $06-12 / 05 / 99$ & 25,0 & 72.675 & 2.907 \\
\hline & BR 35 & $01 / 12 / 98$ & $14 / 05 / 99$ & 10,0 & 27.190 & 2.719 \\
\hline & - & - & - & - & - & - \\
\hline \multirow{3}{*}{ 1999/00 } & BR 133 & $22-23 / 12 / 99$ & $22-23 / 05 / 00$ & 22,0 & 59.444 & 2.702 \\
\hline & $\begin{array}{c}\text { EMBRAPA } 59 \\
\text { CD } 205\end{array}$ & $\begin{array}{l}29-30 / 12 / 99 \\
16-19 / 12 / 99\end{array}$ & $\begin{array}{l}24-25 / 05 / 00 \\
30-31 / 05 / 00\end{array}$ & $\begin{array}{l}13,0 \\
250\end{array}$ & 28.977 & $\begin{array}{l}2.229 \\
2449\end{array}$ \\
\hline & EMBRAPA 60 & $01-02 / 12 / 99$ & $27 / 04$ a- 3/05/00 & $\frac{3,0}{38,0}$ & 90.288 & $\frac{2.449}{2.376}$ \\
\hline \multirow{2}{*}{ 2000/01 } & BR 133 & $\begin{array}{c}24 / 11 /- \\
07 / 12 / 00\end{array}$ & 01/05-18/05/01 & 61,0 & 181.515 & 2.976 \\
\hline & CD 205 & $\begin{array}{c}24 / 11- \\
07 / 12 / 00\end{array}$ & 01/05-18/05/01 & 64,2 & 191.205 & 2.978 \\
\hline
\end{tabular}

Fonte: Banco de Dados da Coordenação Geral da Fazenda Experimental Gralha Azul da PUCPR (2001)

Para cinco intervalos escolhidos, de maturação da soja, a partir do Banco de Dados Hidrológicos, da Estação Pluviográfica Curitiba Prado Velho, as alturas pluviométricas acumuladas estão indicadas na Tabela 2. 
TABELA 2 - Alturas pluviométricas acumuladas (mm)

Table 2-Accumulated pluvial heights ( $\mathrm{mm}$ )

\begin{tabular}{cc|c|ccc}
\hline Ano & $\mathrm{I}_{(45-75)}$ & $\mathrm{I}_{(45-90)}$ & $\mathrm{I}_{(75-90)}$ & $\mathrm{I}_{(30-120)}$ & $\mathrm{I}_{(0-120)}$ \\
\hline $1993 / 94$ & 326 & 393 & 82 & 515 & 584 \\
$1994 / 95$ & 423 & 523 & 99 & 736 & 905 \\
$1995 / 96$ & 282 & 389 & 143 & 792 & 841 \\
$1996 / 97$ & 378 & 588 & 218 & 826 & 955 \\
$1997 / 98$ & 133 & 188 & 79 & 641 & 811 \\
$1998 / 99$ & 379 & 385 & 8 & 682 & 885 \\
\hline $1999 / 00$ & 210 & 258 & 107 & 420 & 544 \\
$2000 / 01$ & 284 & 301 & 95 & 814 & 951 \\
\hline
\end{tabular}

Fonte: Banco de Dados Hidrológicos do Laboratório de Climatologia, Hidrologia e Recursos Hídricos do Curso de Engenharia Civil da PUCPR (2001)

Das Tabelas 1 e 2, extraíram-se os valores para o estabelecimento das correlações entre a produtividade da soja e as alturas pluviométricas acumuladas, as quais estão indicadas na Tabela 3.

TABELA 3 - Valores da produtividade da soja e alturas pluviométricas acumuladas Table 3-Soybean productivity and accumulated pluvial heights

\begin{tabular}{|c|c|c|c|c|c|c|c|}
\hline \multirow{2}{*}{ Ano } & \multirow{2}{*}{$\mathbf{N}$} & \multirow{2}{*}{$\begin{array}{l}\text { Produtividade } \\
\text { (kg/ha) }\end{array}$} & \multicolumn{5}{|c|}{ Precipitações Acumuladas (mm) } \\
\hline & & & $\mathbf{I}_{(45-75)}$ & $\mathbf{I}_{(45-90)}$ & $I_{(75-90)}$ & $I_{(30-120)}$ & $I_{(0-120)}$ \\
\hline $1993 / 94$ & 1 & 2.716 & 326 & 393 & 82 & 515 & 584 \\
\hline $1994 / 95$ & 2 & 2.949 & 423 & 523 & 99 & 736 & 905 \\
\hline $1995 / 96$ & 3 & 2.134 & 282 & 389 & 173 & 792 & 841 \\
\hline $1996 / 97$ & 4 & 2.159 & 378 & 588 & 218 & 826 & 955 \\
\hline $1997 / 98$ & 5 & 2.271 & 133 & 188 & 79 & 641 & 811 \\
\hline $1998 / 99$ & 6 & 2.813 & 379 & 385 & 8 & 682 & 885 \\
\hline 1999/00 & 7 & 2.439 & 210 & 258 & 107 & 420 & 544 \\
\hline 2000/01 & 8 & 2.977 & 284 & 301 & 95 & 814 & 951 \\
\hline
\end{tabular}


Adotando a metodologia descrita e aplicando-se as Equações (1) a (5), obtiveram-se as seguintes relações entre a produtividade da soja e alturas pluviais acumuladas na Fazenda Experimental Gralha Azul da PUCPR:

$$
\begin{aligned}
& \mathrm{Pe}=2.086,74+1,55 \mathrm{I}(45-75) \\
& \mathrm{Pe}=2.584,96+0,07 \mathrm{I}(45-90) \\
& \mathrm{Pe}=2.946,43+3,61 \mathrm{I}(75-90) \\
& \mathrm{Pe}=2.606,76-0,07 \mathrm{I}(30-120) \\
& \mathrm{Pe}=2.375,67+0,22 \mathrm{I}(0-120)
\end{aligned}
$$

Onde: $\mathrm{Pe}=$ Produtividade Média Esperada da Cultura da Soja (kg/ha);

I( $i-j)=$ Precipitação pluvial acumulada no período do ciclo vegetativo da cultura $(\mathrm{mm})$

Com as relações obtidas nas Equações (6) a (10), determinadas para cada período do ciclo vegetativo da cultura da soja, na Fazenda Experimental Gralha Azul da PUCPR, calculou-se a produtividade média esperada, indicada nas Tabelas 4 a 8.

TABELA 4 - Produtividade média esperada na cultura da soja no período de (45-75) dias do ciclo vegetativo Table 4 - Waited average productivity in the soybean crop in the period of (45-75) days of the vegetative cycle

\begin{tabular}{|c|c|c|c|}
\hline ANO & $\begin{array}{c}P \\
(\mathrm{~kg} / \mathrm{ha})\end{array}$ & $\begin{array}{c}P_{(45-90)} \\
(\mathrm{kg} / \mathrm{ha})\end{array}$ & $\begin{array}{c}\mathbf{I}_{(45-90)} \\
(\mathbf{m m})\end{array}$ \\
\hline $1993 / 94$ & 2.716 & $2.612,47$ & 393 \\
\hline $1194 / 95$ & 2.949 & $2.621,57$ & 523 \\
\hline $1995 / 96$ & 2.134 & $2.612,19$ & 389 \\
\hline $1996 / 97$ & 2.159 & $2.626,12$ & 588 \\
\hline $1997 / 98$ & 2.271 & $2.598,12$ & 188 \\
\hline $1998 / 99$ & 2.813 & $2.611,91$ & 385 \\
\hline $1999 / 00$ & 2.439 & $2.603,02$ & 258 \\
\hline $2000 / 01$ & 2.977 & $2.606,03$ & 301 \\
\hline MÉDIA & 2.634 & $2.611,49$ & 378 \\
\hline
\end{tabular}

\begin{tabular}{cccc}
\hline ANO & $\begin{array}{c}\mathbf{P} \\
(\mathbf{k g} / \mathbf{h a})\end{array}$ & $\begin{array}{c}\mathbf{P} \mathbf{e}_{(\mathbf{4 5}-\mathbf{7 5})} \\
(\mathbf{k g} / \mathbf{h a})\end{array}$ & $\begin{array}{c}\mathbf{I}_{\mathbf{( 4 5}-\mathbf{7 5})} \\
(\mathbf{m m})\end{array}$ \\
\hline $1993 / 94$ & 2.716 & $2.592,04$ & 326 \\
\hline $1994 / 95$ & 2.949 & $2.742,39$ & 423 \\
$1995 / 96$ & 2.134 & $2.523,84$ & 282 \\
$1996 / 97$ & 2.159 & $2.672,64$ & 378 \\
\hline $1997 / 98$ & 2.271 & $2.292,89$ & 133 \\
\hline $1998 / 99$ & 2.813 & $2.674,19$ & 379 \\
\hline $1999 / 00$ & 2.439 & $2.412,24$ & 210 \\
\hline 2000 / O1 & 2.977 & $2.526,94$ & 302 \\
\hline MEDIA & 2.634 & $2.554,65$ & 302 \\
\hline
\end{tabular}

TABELA 5 - Produtividade média esperada na cultura da soja, no período de (45-90) dias do ciclo vegetativo Table 5 - Waited average productivity in the soybean crop in the period of (45-90) days of the vegetative cycle 
TABELA 6 - Produtividade média esperada na cultura da soja no período de (75-90) dias do ciclo vegetativo Table 6 - Waited average productivity in the soybean crop in the period of (75-90) days of the vegetative cycle

\begin{tabular}{|c|c|c|c|}
\hline ANO & $\begin{array}{c}\mathbf{P} \\
(\mathrm{kg} / \mathrm{ha})\end{array}$ & $\begin{array}{c}\text { Pe }_{(75-90)} \\
(\mathrm{kg} / \mathrm{ha})\end{array}$ & $\begin{array}{c}\mathbf{I}_{(75-90)} \\
(\mathbf{m m})\end{array}$ \\
\hline 1993 / 94 & 2.716 & $3.242,45$ & 82 \\
\hline 1994 / 95 & 2.949 & $3.303,82$ & 99 \\
\hline $1995 / 96$ & 2.134 & $3.570,96$ & 173 \\
\hline $1996 / 97$ & 2.159 & $3.733,41$ & 218 \\
\hline 1997 / 98 & 2.271 & $3.231,62$ & 79 \\
\hline 1998 / 99 & 2.813 & $2.975,31$ & 8 \\
\hline 1999 / 00 & 2.439 & $3.332,70$ & 107 \\
\hline $2000 / 01$ & 2.977 & $3.289,38$ & 95 \\
\hline MEDDIA & 2.634 & $3.334,96$ & 108 \\
\hline
\end{tabular}

TABELA 7 - Produtividade média esperada na cultura da soja, no período de (30-120) dias do ciclo vegetativo Table 7 - Waited average productivity in the soybean crop in the period of (30-120) days of the vegetative cycle

\begin{tabular}{|c|c|c|c|}
\hline ANO & $\begin{array}{c}\mathbf{P} \\
(\mathbf{K g} / \mathbf{h a})\end{array}$ & $\begin{array}{c}P \mathbf{e}_{(30-120)} \\
(\mathbf{K g} / \mathbf{h a})\end{array}$ & $\begin{array}{c}\mathbf{I}_{(\mathbf{3 0}-\mathbf{1 2 0})} \\
(\mathbf{m m})\end{array}$ \\
\hline 1993 / 94 & 2.716 & $2.570,71$ & 515 \\
\hline 1994 / 95 & 2.949 & $2.555,24$ & 736 \\
\hline 1995 / 96 & 2.134 & $2.551,32$ & 792 \\
\hline $1996 / 97$ & 2.159 & $2.548,94$ & 826 \\
\hline 1997 / 98 & 2.271 & $2.561,89$ & 641 \\
\hline $1998 / 99$ & 2.813 & $2.559,02$ & 682 \\
\hline $1999 / 00$ & 2.439 & $2.577,36$ & 420 \\
\hline $2000 / 01$ & 2.977 & $2.549,78$ & 814 \\
\hline MEDIA & 2.634 & $2.559,28$ & 678 \\
\hline
\end{tabular}

TABELA 8 - Produtividade media esperada na cultura da soja, no período de (0-120) dias do ciclo vegetativo. Table 8 - Waited average productivity in the soybean crop in the period of (0-120) days of the vegetative cycle

\begin{tabular}{cccc}
\hline ANO & $\begin{array}{c}\mathbf{P} \\
\mathbf{( k g / h a )}\end{array}$ & $\begin{array}{c}\mathbf{P e}_{(\mathbf{0}-\mathbf{1 2 0})} \\
(\mathbf{k g} / \mathbf{h a})\end{array}$ & $\begin{array}{c}\mathbf{I}_{(\mathbf{0}-\mathbf{1 2 0})} \\
(\mathbf{m m})\end{array}$ \\
\hline $1993 / 94$ & 2.716 & $2.504,15$ & 584 \\
\hline $1994 / 95$ & 2.949 & $2.574,77$ & 905 \\
$1995 / 96$ & 2.134 & $2.560,69$ & 841 \\
$1996 / 97$ & 2.159 & $2.585,77$ & 955 \\
\hline $1997 / 98$ & 2.271 & $2.554,09$ & 811 \\
$1998 / 99$ & 2.813 & $2.570,37$ & 885 \\
$1999 / 00$ & 2.439 & $2.495,35$ & 544 \\
\hline $2000 / 01$ & 2.977 & $2.584,89$ & 951 \\
\hline MÉDIA & 2.634 & $2.553,76$ & 809
\end{tabular}




\section{Análise dos Resultados Obtidos}

De acordo as relações obtidas nas Equações (6) a (10), observamos que:

A disponibilidade de água é importante, principalmente, em dois períodos de desenvolvimento da soja: germinação/emergência e na floração/enchimento de grãos. Durante o primeiro período, tanto o excesso quanto o déficit de água são prejudiciais à obtenção de uma boa uniformidade na população de plantas. A semente de soja necessita absorver, no mínimo, 50\% de seu peso em água para assegurar uma boa germinação. Nesta fase, o conteúdo de água no solo não deve exceder a 85\% do total de água disponível e nem ser inferior a 50\%. Pelas Tabelas 4 a 8 , visualiza-se que nos respectivos períodos de desenvolvimento da soja: $(45-75),(45-90),(30-120)$ e $(0-120)$ dias, a produtividade média obtida na Fazenda Experimental Gralha Azul nos anos de 1994, 1995, 1999 e 2001 revelou-se acima da produtividade média esperada, determinada pela metodologia de Tubelis ( 1988). Apenas no menor período de tempo da chuva acumulada no período de $(75-90)$ dias, Tabela 6, essa produtividade obtida revelouse abaixo da produtividade esperada.

A necessidade de água na cultura da soja aumenta com o desenvolvimento da planta, atingindo o máximo durante a floração/enchimento de grãos ( 7 a $8 \mathrm{~mm} / \mathrm{dia}$ ), decrescendo após este período. Déficit hídrico expressivo, durante a floração/ enchimento de grãos, provoca alterações fisiológicas na planta, como o fechamento dos estômatos e enrolamento de folhas e, como conseqüência, causam a queda prematura de folhas, queda de flores e abortamento de vagens, resultando, por fim, na redução do rendimento de grãos. Considerando a máxima necessidade de água da chuva $\cong 7 \mathrm{~mm} /$ dia, para o período de floração/enchimento de grãos da cultura de soja, totalizando $840 \mathrm{~mm}$ no período (0-120) dias, observa-se, do que consta na Tabela 8, que nos anos de 1996 (841mm) e 1997 (955 mm) a produtividade média obtida revelou-se abaixo da produtividade média esperada.

Para obtenção do rendimento máximo, a necessidade de água na cultura da soja, durante todo o seu ciclo, varia entre 450 a $800 \mathrm{~mm}$, dependendo das condições climáticas, do manejo da cultura e da duração do seu ciclo, o que pode ser constatado na Tabela 8, para o período (0-120) dias do ciclo vegetativo da cultura.

\section{Conclusões e recomendações}

De acordo com as observações de campo e relações obtidas nas Equações (6) a (10) e Tabelas 4 a 8, conclui-se que:

- A disponibilidade de água é importante, principalmente, em dois períodos de desenvolvimento da soja: germinação/emergência e na floração/enchimento dos grãos. Durante o primeiro período, tanto o excesso quanto o déficit de água são prejudiciais à obtenção de uma boa uniformidade na população de plantas. A semente da soja necessita absorver, no mínimo, 50\% de seu peso em água para assegurar uma boa germinação. Nesta fase, o conteúdo de água no solo não deve exceder a $85 \%$ do total de água disponível e nem ser inferior a $50 \%$;

- A necessidade de água na cultura da soja vai aumentando com o desenvolvimento da planta, atingindo o máximo durante a floração/ enchimento dos grãos (7 a $8 \mathrm{~mm} / \mathrm{dia}$ ), decrescendo após este período. Déficits hídricos expressivos durante a floração/enchimento de grãos provocam alterações fisiológicas na planta, como o fechamento dos estômatos e o enrolamento das folhas e, como conseqüência, causam a queda prematura das folhas, queda de flores e abortamento das vagens, resultando, por fim, na redução do rendimento dos grãos;

- Para obtenção do rendimento máximo, a necessidade de água na cultura da soja, durante todo o seu ciclo, varia entre 450 a $800 \mathrm{~mm}$, dependendo das condições climáticas, do manejo da cultura e da duração do seu ciclo;

- A cultura da soja, cultivada na região do Município de Fazenda Rio Grande, PR, mais precisamente na Fazenda Experimental Gralha Azul da PUCPR, não necessita de suplementação hídrica, pois, a precipitação regional supre a necessidade da cultura, estabelecendo elevados índices de produtividade, se comparado com a produtividade esperada, quando aplicada a equação da produtividade, e a produtividade média obtida no Estado do Paraná, conforme indicado na Tabela 9 e visualizada na Figura 2. 
TABELA 9 - Comparativo da produtividade média da soja na Fazenda Experimental Gralha Azul da PUCPR x Estado do Paraná

Table 9 - Comparative degree of the average soybean productivity in the Experimental Farm Gralha Azul of PUCPR $x$ State of Parana

\begin{tabular}{c|c|c|c}
\hline ANO & $\begin{array}{c}\mathbf{P} \\
\mathbf{( k g / h a )}\end{array}$ & $\begin{array}{c}\mathbf{P e}_{\mathbf{( 0 - 1 2 0}} \\
\mathbf{( k g / h a )}\end{array}$ & $\begin{array}{c}\mathbf{P} \text { PR } \\
\mathbf{( k g / h a )}\end{array}$ \\
$1993 / 94$ & 2.716 & $2.504,15$ & $2.475,72$ \\
$1994 / 95$ & 2.949 & $2.574,77$ & $2.581,04$ \\
\hline $1995 / 96$ & 2.134 & $2.560,69$ & $2.698,63$ \\
$1996 / 97$ & 2.159 & $2.585,77$ & $2.591,44$ \\
\hline $1997 / 98$ & 2.271 & $2.554,09$ & $2.558,32$ \\
$1998 / 99$ & 2.813 & $2.570,37$ & $2.781,80$ \\
\hline $1999 / 00$ & 2.439 & $2.495,35$ & $2.510,92$ \\
$2000 / 01$ & 2.977 & $2.584,89$ & $2.949,87$ \\
MÉDIA & 2.634 & $2.553,76$ & $2.643,47$
\end{tabular}

$\mathrm{P}=$ Produtividade Média da Cultura da Soja na Fazenda Experimental Gralha Azul da PUCPR

$\mathrm{Pe}=$ Produtividade Média Esperada da Cultura da Soja

$\mathrm{P}_{\mathrm{PR}}=$ Produtividade Média da Cultura da Soja no Estado do Paraná

FIGURA 2 - Produtividade média da cultura da soja na Fazenda Experimental Gralha Azul x Estado do Paraná

Figure 2 - Average Productivity of the soybean crop in the Experimental Farm Gralha Azul x State

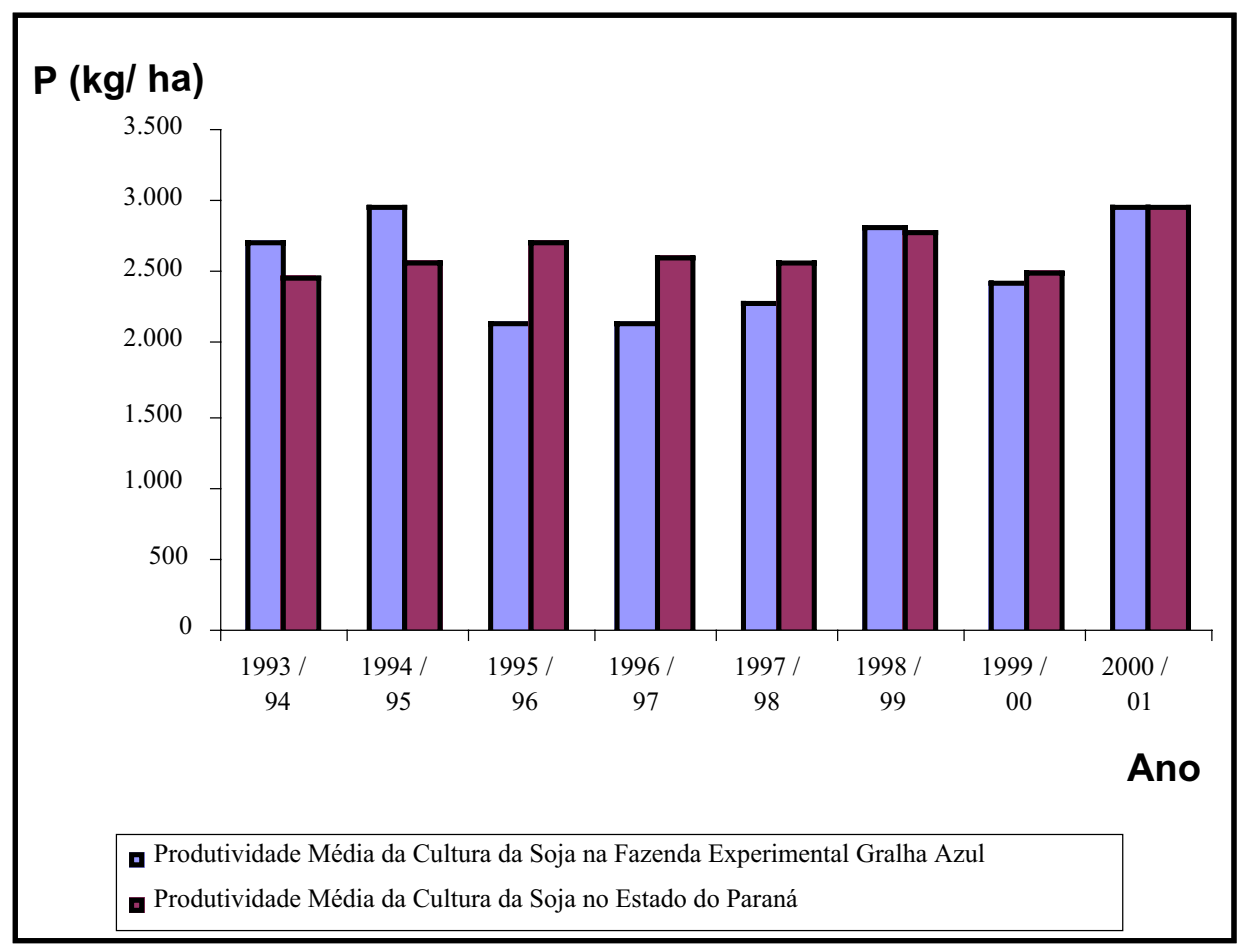


- Da análise dos dados da Tabela 9 e da representação na Figura 2, para o período de 1994 - 2001, observa-se que ocorreram oscilações na produtividade da soja entre a Fazenda Experimental Gralha Azul da PUCPR e o Estado do Paraná, não por conseqüência de chuvas abundantes, normais ou de "Déficit" hídrico, e sim, exclusivamente, por questões do manejo da cultura da soja. Da Tabela 9 e da Figura 2, conclui-se que não há necessidade de se fazer sistema de irrigação na cultura da soja, na Fazenda Experimental Gralha Azul da PUCPR, quando a precipitação pluvial acumulada no período vegetativo da cultura de $(0-$ 120) dias for superior a $600 \mathrm{~mm}$. A cultura da soja apesar de apresentar grande demanda hídrica para o seu desenvolvimento, também tem capacidade de adaptação a "déficit" de água, como ocorrido nos anos de 1994 (584 mm) e 2000 ( $544 \mathrm{~mm}$ ), respectivamente;

- Do comparativo entre a produtividade média obtida da cultura da soja na Fazenda Experimental Gralha Azul e no Estado do Paraná, emana a recomendação de que é importante o ade- quado manejo da cultura, com aplicação da conservação dos solos e água, escolha de cultivar adaptada à região, adequada população de plantas cultivadas, controle de pragas e doenças, entre outras.

\section{Referências}

FENDRICH, R. Relações Entre a Chuva e a Produtividade da Soja na Fazenda Experimental Gralha Azul da PUCPR. Curso de Agronomia, Fazenda Rio Grande - PR, 2001. 40p.

GOMES, P. A. Soja. Editora Nobel, São Paulo - SP, 5. ed., 1990. 152p.

TUBelis, A. A Chuva e a Produção Agrícola. Editora Nobel S.A., São Paulo - SP, 1988. 85p

Recebido 30/6/2002

Aprovado 3/3/2003 